\title{
Quantum-Chemical Analysis of Hetacillin Molecule via DFT and AB INITIO Methods
}

\author{
Babkin V.A. ${ }^{1, *}$ Ignatov A.V. ${ }^{1}$ Reshetnikova M.V. ${ }^{2}$ Andreev D.S. ${ }^{1}$ Liberovskaya A.N. ${ }^{2}$ \\ Belousova V.S. ${ }^{3}$ Rakhimov A.I. ${ }^{4}$ Titova E.S. ${ }^{4}$ \\ ${ }^{1}$ Department of Mathematics and Natural Sciences, Sebraykov Branch of Volgograd State Technical University, \\ Mikhailovka, Russia \\ ${ }^{2}$ Department of Technical Disciplines and Thermal Power Engineering, Sebraykov Branch of Volgograd State Technical \\ University, Mikhailovka, Russia \\ ${ }^{3}$ Department of Obstetrics, Gynecology and Perinatology, First Moscow State Medical University named after \\ I.M. Sechenov, Moscow, Russia \\ ${ }^{4}$ Department of Organic Chemistry, Volgograd State Technical University, Volgograd, Russia \\ *Corresponding author. Email: babkin_v.a@mail.ru
}

\section{ABSTRACT}

For the first time quantum-chemical calculation of $\beta$-lactam antibiotic hetacillin molecule via DFT and $\mathrm{AB}$ INITIO methods with the optimization of geometry of all parameters by standard gradient method was performed. An optimized geometric and electronic structure of this compound (optimized bond lengths, valency angles and atomic charges) was obtained, and the total energy of the molecule was calculated. Its acid strength is theoretically estimated. It was found that the hetacillin molecule belongs to the class of weak $\mathrm{OH}$ acids.

Keywords: quantum-chemical calculation, AB INITIO method, DFT method, hetacillin, acid strength

\section{INTRODUCTION}

Hetacillin is a $\beta$-lactam antibiotic that is part of the aminopenicillin family. It is a prodrug that does not have antibacterial activity in itself, but quickly breaks down acetone in the human body to form ampicillin, which is active against various bacteria [6].

According to IUPAC nomenclature, hetacillin has the following name: (2S,5R,6R)-6-[(4R)-2.2-dimetyl-5-oxo 4- phenylidazolidin-1-yl]-3.3 - dimethyl-7-oxo-4-thia1azabicyclo[3.2.0]heptane-2-carboxylic acid.

Figure 1 shows the structural formula of Hetacillin.

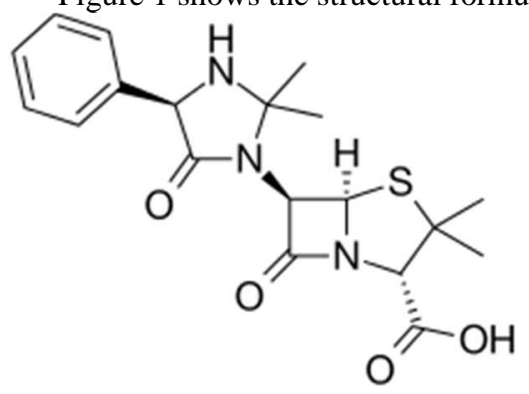

Figure 1 Structural formula of hetacillin

As can be seen from the structure of hetacillin, it belongs to $\beta$-lactam antibiotics.

The purpose of the study is the quantum-chemical analysis of the hetacillin molecule via DFT and $A B$ INITIO methods.

\section{METHODS AND MATERIALS}

We calculated the hetacillin molecule by DFT-PBE0/6$311 \mathrm{G}^{* *}$ and $\mathrm{AB}$ INITIO/6-311G** quantum-chemical methods with the optimization of geometry regarding all parameters by the standard gradient method built into Firefly [4], which is partly based on the source code GAMESS (US) [3] and in the approximation of the isolated molecule in gaseous phase and theoretical assessment of its acid strength. A known MacMolPlt program [5] was used to visualize the molecule model.

\section{RESULTS}

The optimized geometrical and electronic structure and the total energy of the hetacillin molecule were obtained via DFT-PBE0/6-311G** and AB INITIO/6-311G** methods and are shown in Figures 2-3. Tables 1-2 show the optimized bond lengths, valency angles and atomic charges of the hetacillin molecule by the above quantum chemistry methods. Using the formulas $\mathrm{pKa}=51.048$ $150.078 \mathrm{q}_{\max }{ }^{\mathrm{H}+}$ [2] (DFT method, $\mathrm{q}_{\max }{ }^{\mathrm{H}+}=+0.26-$ maximum charge on hydrogen atom, pKa - universal acidity index, Table 1) and $\mathrm{pKa}=49.04-134.61 \mathrm{q}_{\max }^{\mathrm{H}+}[1]$ (AB INITIO method, $\mathrm{q}_{\max }{ }^{\mathrm{H}+}=+0.28$, Table 2), we find values of acid strength equal $\mathrm{pKa}=12$ and 11 , respectively. 
Table 1 Optimized bond lengths, valency angles and atomic charges of the hetacillin molecule via DFT method

\begin{tabular}{|c|c|c|c|c|c|}
\hline Bond lengths & $\mathbf{R}, \mathbf{A}$ & Valency angles & Deg & Atom & Atomic charges \\
\hline $\mathrm{C}(2)-\mathrm{C}(1)$ & 1.39 & $\mathrm{C}(6)-\mathrm{C}(5)-\mathrm{C}(1)$ & 120 & $\mathrm{C}(1)$ & -0.11 \\
\hline $\mathrm{C}(3)-\mathrm{C}(2)$ & 1.39 & $\mathrm{C}(1)-\mathrm{C}(2)-\mathrm{C}(3)$ & 121 & $\mathrm{C}(2)$ & -0.02 \\
\hline $\mathrm{C}(4)-\mathrm{C}(3)$ & 1.40 & $\mathrm{C}(2)-\mathrm{C}(3)-\mathrm{C}(4)$ & 119 & $\mathrm{C}(3)$ & -0.15 \\
\hline $\mathrm{C}(5)-\mathrm{C}(1)$ & 1.39 & $\mathrm{C}(7)-\mathrm{C}(3)-\mathrm{C}(4)$ & 121 & $\mathrm{C}(4)$ & -0.03 \\
\hline $\mathrm{C}(5)-\mathrm{C}(6)$ & 1.39 & $\mathrm{C}(2)-\mathrm{C}(1)-\mathrm{C}(5)$ & 120 & $\mathrm{C}(5)$ & -0.08 \\
\hline $\mathrm{C}(6)-\mathrm{C}(4)$ & 1.39 & $\mathrm{C}(4)-\mathrm{C}(6)-\mathrm{C}(5)$ & 120 & $\mathrm{C}(6)$ & -0.13 \\
\hline $\mathrm{C}(7)-\mathrm{C}(3)$ & 1.50 & $\mathrm{C}(3)-\mathrm{C}(4)-\mathrm{C}(6)$ & 120 & $\mathrm{C}(7)$ & -0.10 \\
\hline $\mathrm{C}(8)-\mathrm{C}(7)$ & 1.53 & $\mathrm{C}(2)-\mathrm{C}(3)-\mathrm{C}(7)$ & 120 & $\mathrm{C}(8)$ & +0.45 \\
\hline $\mathrm{O}(9)-\mathrm{C}(8)$ & 1.21 & $\mathrm{C}(3)-\mathrm{C}(7)-\mathrm{C}(8)$ & 115 & $\mathrm{O}(9)$ & -0.38 \\
\hline $\mathrm{N}(10)-\mathrm{C}(8)$ & 1.37 & $\mathrm{~N}(39)-\mathrm{C}(7)-\mathrm{C}(8)$ & 105 & $\mathrm{~N}(10)$ & -0.39 \\
\hline $\mathrm{C}(11)-\mathrm{N}(10)$ & 1.42 & $\mathrm{C}(7)-\mathrm{C}(8)-\mathrm{O}(9)$ & 127 & $\mathrm{C}(11)$ & -0.13 \\
\hline $\mathrm{C}(11)-\mathrm{C}(12)$ & 1.56 & $\mathrm{~N}(10)-\mathrm{C}(8)-\mathrm{O}(9)$ & 127 & $\mathrm{C}(12)$ & -0.12 \\
\hline $\mathrm{C}(12)-\mathrm{N}(14)$ & 1.45 & $\mathrm{C}(7)-\mathrm{C}(8)-\mathrm{N}(10)$ & 106 & $\mathrm{C}(13)$ & +0.45 \\
\hline $\mathrm{C}(13)-\mathrm{C}(11)$ & 1.56 & $\mathrm{C}(12)-\mathrm{C}(11)-\mathrm{N}(10)$ & 122 & $\mathrm{~N}(14)$ & -0.33 \\
\hline $\mathrm{N}(14)-\mathrm{C}(13)$ & 1.39 & $\mathrm{~N}(39)-\mathrm{C}(42)-\mathrm{N}(10)$ & 103 & $\mathrm{O}(15)$ & -0.33 \\
\hline $\mathrm{O}(15)-\mathrm{C}(13)$ & 1.19 & $\mathrm{C}(8)-\mathrm{N}(10)-\mathrm{C}(11)$ & 124 & $\mathrm{~S}(16)$ & +0.15 \\
\hline $\mathrm{S}(16)-\mathrm{C}(12)$ & 1.82 & $\mathrm{~N}(14)-\mathrm{C}(12)-\mathrm{C}(11)$ & 88 & $\mathrm{C}(17)$ & -0.07 \\
\hline $\mathrm{C}(17)-\mathrm{N}(14)$ & 1.44 & $\mathrm{~S}(16)-\mathrm{C}(12)-\mathrm{C}(11)$ & 121 & $\mathrm{C}(18)$ & +0.41 \\
\hline $\mathrm{C}(18)-\mathrm{C}(17)$ & 1.52 & $\mathrm{C}(13)-\mathrm{N}(14)-\mathrm{C}(12)$ & 95 & $\mathrm{O}(19)$ & -0.31 \\
\hline $\mathrm{O}(19)-\mathrm{C}(18)$ & 1.34 & $\mathrm{~N}(10)-\mathrm{C}(11)-\mathrm{C}(13)$ & 121 & $\mathrm{O}(20)$ & -0.35 \\
\hline $\mathrm{O}(20)-\mathrm{C}(18)$ & 1.20 & $\mathrm{C}(12)-\mathrm{C}(11)-\mathrm{C}(13)$ & 85 & $\mathrm{H}(21)$ & +0.26 \\
\hline $\mathrm{H}(21)-\mathrm{O}(19)$ & 0.97 & $\mathrm{C}(11)-\mathrm{C}(13)-\mathrm{N}(14)$ & 90 & $\mathrm{C}(22)$ & -0.44 \\
\hline $\mathrm{C}(22)-\mathrm{C}(17)$ & 1.58 & $\mathrm{O}(15)-\mathrm{C}(13)-\mathrm{N}(14)$ & 132 & $\mathrm{C}(23)$ & -0.24 \\
\hline $\mathrm{C}(22)-\mathrm{S}(16)$ & 1.87 & $\mathrm{C}(11)-\mathrm{C}(13)-\mathrm{O}(15)$ & 137 & $\mathrm{C}(24)$ & -0.27 \\
\hline $\mathrm{C}(23)-\mathrm{C}(22)$ & 1.53 & $\mathrm{~N}(14)-\mathrm{C}(12)-\mathrm{S}(16)$ & 106 & $\mathrm{H}(25)$ & +0.18 \\
\hline $\mathrm{C}(24)-\mathrm{C}(22)$ & 1.53 & $\mathrm{C}(17)-\mathrm{C}(22)-\mathrm{S}(16)$ & 105 & $\mathrm{H}(26)$ & +0.13 \\
\hline $\mathrm{H}(25)-\mathrm{C}(23)$ & 1.09 & $\mathrm{C}(13)-\mathrm{N}(14)-\mathrm{C}(17)$ & 129 & $\mathrm{H}(27)$ & +0.13 \\
\hline $\mathrm{H}(26)-\mathrm{C}(23)$ & 1.10 & $\mathrm{~N}(14)-\mathrm{C}(17)-\mathrm{C}(18)$ & 113 & $\mathrm{H}(28)$ & +0.15 \\
\hline $\mathrm{H}(27)-\mathrm{C}(23)$ & 1.09 & $\mathrm{C}(22)-\mathrm{C}(17)-\mathrm{C}(18)$ & 113 & $\mathrm{H}(29)$ & +0.14 \\
\hline $\mathrm{H}(28)-\mathrm{C}(24)$ & 1.09 & $\mathrm{C}(17)-\mathrm{C}(18)-\mathrm{O}(19)$ & 113 & $\mathrm{H}(30)$ & +0.14 \\
\hline $\mathrm{H}(29)-\mathrm{C}(24)$ & 1.09 & $\mathrm{O}(20)-\mathrm{C}(18)-\mathrm{O}(19)$ & 123 & $\mathrm{H}(31)$ & +0.10 \\
\hline $\mathrm{H}(30)-\mathrm{C}(24)$ & 1.09 & $\mathrm{C}(17)-\mathrm{C}(18)-\mathrm{O}(20)$ & 124 & $\mathrm{H}(32)$ & +0.10 \\
\hline $\mathrm{H}(31)-\mathrm{C}(5)$ & 1.09 & $\mathrm{C}(18)-\mathrm{O}(19)-\mathrm{H}(21)$ & 106 & $\mathrm{H}(33)$ & +0.13 \\
\hline $\mathrm{H}(32)-\mathrm{C}(6)$ & 1.09 & $\mathrm{~N}(14)-\mathrm{C}(17)-\mathrm{C}(22)$ & 108 & $\mathrm{H}(34)$ & +0.10 \\
\hline $\mathrm{H}(33)-\mathrm{C}(4)$ & 1.09 & $\mathrm{C}(12)-\mathrm{S}(16)-\mathrm{C}(22)$ & 95 & $\mathrm{H}(35)$ & +0.10 \\
\hline $\mathrm{H}(34)-\mathrm{C}(1)$ & 1.09 & $\mathrm{C}(17)-\mathrm{C}(22)-\mathrm{C}(23)$ & 109 & $\mathrm{H}(36)$ & +0.18 \\
\hline $\mathrm{H}(35)-\mathrm{C}(2)$ & 1.09 & $\mathrm{~S}(16)-\mathrm{C}(22)-\mathrm{C}(23)$ & 110 & $\mathrm{H}(37)$ & +0.20 \\
\hline $\mathrm{H}(36)-\mathrm{C}(11)$ & 1.09 & $\mathrm{C}(24)-\mathrm{C}(22)-\mathrm{C}(23)$ & 111 & $\mathrm{H}(38)$ & +0.18 \\
\hline $\mathrm{H}(37)-\mathrm{C}(17)$ & 1.09 & $\mathrm{C}(17)-\mathrm{C}(22)-\mathrm{C}(24)$ & 113 & $\mathrm{~N}(39)$ & -0.35 \\
\hline $\mathrm{H}(38)-\mathrm{C}(12)$ & 1.09 & $\mathrm{~S}(16)-\mathrm{C}(22)-\mathrm{C}(24)$ & 108 & $\mathrm{H}(40)$ & +0.17 \\
\hline $\mathrm{N}(39)-\mathrm{C}(7)$ & 1.47 & $\mathrm{C}(22)-\mathrm{C}(23)-\mathrm{H}(25)$ & 112 & $\mathrm{H}(41)$ & +0.21 \\
\hline $\mathrm{H}(40)-\mathrm{C}(7)$ & 1.10 & $\mathrm{C}(22)-\mathrm{C}(23)-\mathrm{H}(26)$ & 110 & $\mathrm{C}(42)$ & -0.05 \\
\hline $\mathrm{H}(41)-\mathrm{N}(39)$ & 1.02 & $\mathrm{C}(22)-\mathrm{C}(23)-\mathrm{H}(27)$ & 110 & $\mathrm{C}(43)$ & -0.29 \\
\hline $\mathrm{C}(42)-\mathrm{N}(39)$ & 1.46 & $\mathrm{C}(22)-\mathrm{C}(24)-\mathrm{H}(28)$ & 110 & $\mathrm{C}(44)$ & -0.19 \\
\hline $\mathrm{C}(42)-\mathrm{N}(10)$ & 1.48 & $\mathrm{C}(22)-\mathrm{C}(24)-\mathrm{H}(29)$ & 110 & $\mathrm{H}(45)$ & +0.14 \\
\hline $\mathrm{C}(43)-\mathrm{C}(42)$ & 1.52 & $\mathrm{C}(22)-\mathrm{C}(24)-\mathrm{H}(30)$ & 112 & $\mathrm{H}(46)$ & +0.15 \\
\hline $\mathrm{C}(44)-\mathrm{C}(42)$ & 1.53 & $\mathrm{C}(1)-\mathrm{C}(5)-\mathrm{H}(31)$ & 120 & $\mathrm{H}(47)$ & +0.13 \\
\hline $\mathrm{H}(45)-\mathrm{C}(43)$ & 1.09 & $\mathrm{C}(6)-\mathrm{C}(5)-\mathrm{H}(31)$ & 120 & $\mathrm{H}(48)$ & +0.13 \\
\hline $\mathrm{H}(46)-\mathrm{C}(43)$ & 1.09 & $\mathrm{C}(4)-\mathrm{C}(6)-\mathrm{H}(32)$ & 120 & $\mathrm{H}(49)$ & +0.13 \\
\hline $\mathrm{H}(47)-\mathrm{C}(43)$ & 1.09 & $\mathrm{C}(3)-\mathrm{C}(4)-\mathrm{H}(33)$ & 120 & $\mathrm{H}(50)$ & +0.11 \\
\hline $\mathrm{H}(48)-\mathrm{C}(44)$ & 1.09 & $\mathrm{C}(2)-\mathrm{C}(1)-\mathrm{H}(34)$ & 120 & & \\
\hline $\mathrm{H}(49)-\mathrm{C}(44)$ & 1.09 & $\mathrm{C}(1)-\mathrm{C}(2)-\mathrm{H}(35)$ & 120 & & \\
\hline \multirow[t]{8}{*}{$\mathrm{H}(50)-\mathrm{C}(44)$} & 1.09 & $\mathrm{~N}(10)-\mathrm{C}(11)-\mathrm{H}(36)$ & 109 & & \\
\hline & & $\mathrm{C}(12)-\mathrm{C}(11)-\mathrm{H}(36)$ & 109 & & \\
\hline & & $\mathrm{N}(14)-\mathrm{C}(17)-\mathrm{H}(37)$ & 109 & & \\
\hline & & $\mathrm{N}(14)-\mathrm{C}(12)-\mathrm{H}(38)$ & 114 & & \\
\hline & & $\mathrm{C}(3)-\mathrm{C}(7)-\mathrm{N}(39)$ & 112 & & \\
\hline & & $\mathrm{C}(3)-\mathrm{C}(7)-\mathrm{H}(40)$ & 109 & & \\
\hline & & $\mathrm{C}(7)-\mathrm{N}(39)-\mathrm{H}(41)$ & 107 & & \\
\hline & & $\mathrm{C}(7)-\mathrm{N}(39)-\mathrm{C}(42)$ & 107 & & \\
\hline
\end{tabular}




\begin{tabular}{|l|l|l|l|l|l|}
\hline & & $\mathrm{C}(8)-\mathrm{N}(10)-\mathrm{C}(42)$ & 112 & & \\
\hline & & $\mathrm{N}(39)-\mathrm{C}(42)-\mathrm{C}(43)$ & 110 & & \\
\hline & & $\mathrm{N}(10)-\mathrm{C}(42)-\mathrm{C}(43)$ & 111 & & \\
\hline & & $\mathrm{C}(44)-\mathrm{C}(42)-\mathrm{C}(43)$ & 111 & & \\
\hline & & $\mathrm{N}(39)-\mathrm{C}(42)-\mathrm{C}(44)$ & 110 & & \\
\hline & & $\mathrm{N}(10)-\mathrm{C}(42)-\mathrm{C}(44)$ & 110 & & \\
\hline & & $\mathrm{C}(42)-\mathrm{C}(43)-\mathrm{H}(45)$ & 110 & & \\
\hline & & $\mathrm{C}(42)-\mathrm{C}(43)-\mathrm{H}(46)$ & 109 & & \\
\hline & & $\mathrm{C}(42)-\mathrm{C}(43)-\mathrm{H}(47)$ & 112 & & \\
\hline & & $\mathrm{C}(42)-\mathrm{C}(44)-\mathrm{H}(48)$ & 110 & & \\
\hline & & $\mathrm{C}(42)-\mathrm{C}(44)-\mathrm{H}(49)$ & 111 & & \\
\hline
\end{tabular}

Table 2 Optimized bond lengths, valency angles and atomic charges of the hetacillin molecule via AB INITIO method

\begin{tabular}{|c|c|c|c|c|c|}
\hline Bond lengths & $\mathbf{R , A}$ & Valency angles & Deg & Atom & Atomic charges \\
\hline $\mathrm{C}(2)-\mathrm{C}(1)$ & 1.38 & $\mathrm{C}(4)-\mathrm{C}(3)-\mathrm{C}(2)$ & 119 & $\mathrm{C}(1)$ & -0.09 \\
\hline $\mathrm{C}(3)-\mathrm{C}(2)$ & 1.39 & $\mathrm{C}(1)-\mathrm{C}(2)-\mathrm{C}(3)$ & 121 & $\mathrm{C}(2)$ & -0.04 \\
\hline $\mathrm{C}(3)-\mathrm{C}(4)$ & 1.39 & $\mathrm{C}(6)-\mathrm{C}(4)-\mathrm{C}(3)$ & 120 & $\mathrm{C}(3)$ & -0.14 \\
\hline $\mathrm{C}(4)-\mathrm{C}(6)$ & 1.38 & $\mathrm{C}(5)-\mathrm{C}(6)-\mathrm{C}(4)$ & 120 & $\mathrm{C}(4)$ & -0.06 \\
\hline $\mathrm{C}(5)-\mathrm{C}(1)$ & 1.38 & $\mathrm{C}(2)-\mathrm{C}(1)-\mathrm{C}(5)$ & 120 & $\mathrm{C}(5)$ & -0.09 \\
\hline $\mathrm{C}(6)-\mathrm{C}(5)$ & 1.38 & $\mathrm{C}(1)-\mathrm{C}(5)-\mathrm{C}(6)$ & 120 & $\mathrm{C}(6)$ & -0.10 \\
\hline $\mathrm{C}(7)-\mathrm{C}(3)$ & 1.51 & $\mathrm{C}(2)-\mathrm{C}(3)-\mathrm{C}(7)$ & 119 & $\mathrm{C}(7)$ & +0.03 \\
\hline $\mathrm{C}(8)-\mathrm{C}(7)$ & 1.52 & $\mathrm{C}(4)-\mathrm{C}(3)-\mathrm{C}(7)$ & 122 & $\mathrm{C}(8)$ & +0.57 \\
\hline $\mathrm{O}(9)-\mathrm{C}(8)$ & 1.19 & $\mathrm{C}(3)-\mathrm{C}(7)-\mathrm{C}(8)$ & 116 & $\mathrm{O}(9)$ & -0.50 \\
\hline $\mathrm{N}(10)-\mathrm{C}(8)$ & 1.36 & $\mathrm{~N}(39)-\mathrm{C}(7)-\mathrm{C}(8)$ & 105 & $\mathrm{~N}(10)$ & -0.55 \\
\hline $\mathrm{C}(11)-\mathrm{N}(10)$ & 1.43 & $\mathrm{C}(7)-\mathrm{C}(8)-\mathrm{O}(9)$ & 127 & $\mathrm{C}(11)$ & -0.03 \\
\hline $\mathrm{C}(11)-\mathrm{C}(12)$ & 1.56 & $\mathrm{~N}(10)-\mathrm{C}(8)-\mathrm{O}(9)$ & 127 & $\mathrm{C}(12)$ & -0.03 \\
\hline $\mathrm{C}(12)-\mathrm{N}(14)$ & 1.45 & $\mathrm{C}(7)-\mathrm{C}(8)-\mathrm{N}(10)$ & 107 & $\mathrm{C}(13)$ & +0.57 \\
\hline $\mathrm{C}(13)-\mathrm{C}(11)$ & 1.55 & $\mathrm{C}(12)-\mathrm{C}(11)-\mathrm{N}(10)$ & 123 & $\mathrm{~N}(14)$ & -0.44 \\
\hline $\mathrm{N}(14)-\mathrm{C}(13)$ & 1.39 & $\mathrm{~N}(39)-\mathrm{C}(42)-\mathrm{N}(10)$ & 103 & $\mathrm{O}(15)$ & -0.43 \\
\hline $\mathrm{O}(15)-\mathrm{C}(13)$ & 1.17 & $\mathrm{C}(8)-\mathrm{N}(10)-\mathrm{C}(11)$ & 124 & $\mathrm{~S}(16)$ & +0.09 \\
\hline $\mathrm{S}(16)-\mathrm{C}(12)$ & 1.82 & $\mathrm{~N}(14)-\mathrm{C}(12)-\mathrm{C}(11)$ & 88 & $\mathrm{C}(17)$ & 0.00 \\
\hline $\mathrm{C}(17)-\mathrm{N}(14)$ & 1.44 & $\mathrm{~S}(16)-\mathrm{C}(12)-\mathrm{C}(11)$ & 122 & $\mathrm{C}(18)$ & +0.57 \\
\hline $\mathrm{C}(18)-\mathrm{C}(17)$ & 1.52 & $\mathrm{C}(13)-\mathrm{N}(14)-\mathrm{C}(12)$ & 95 & $\mathrm{O}(19)$ & -0.40 \\
\hline $\mathrm{O}(19)-\mathrm{C}(18)$ & 1.33 & $\mathrm{~N}(10)-\mathrm{C}(11)-\mathrm{C}(13)$ & 122 & $\mathrm{O}(20)$ & -0.45 \\
\hline $\mathrm{O}(20)-\mathrm{C}(18)$ & 1.18 & $\mathrm{C}(12)-\mathrm{C}(11)-\mathrm{C}(13)$ & 85 & $\mathrm{H}(21)$ & +0.28 \\
\hline $\mathrm{H}(21)-\mathrm{O}(19)$ & 0.95 & $\mathrm{C}(11)-\mathrm{C}(13)-\mathrm{N}(14)$ & 91 & $\mathrm{C}(22)$ & -0.37 \\
\hline $\mathrm{C}(22)-\mathrm{C}(17)$ & 1.57 & $\mathrm{O}(15)-\mathrm{C}(13)-\mathrm{N}(14)$ & 132 & $\mathrm{C}(23)$ & -0.16 \\
\hline $\mathrm{C}(22)-\mathrm{S}(16)$ & 1.86 & $\mathrm{C}(11)-\mathrm{C}(13)-\mathrm{O}(15)$ & 137 & $\mathrm{C}(24)$ & -0.19 \\
\hline $\mathrm{C}(23)-\mathrm{C}(22)$ & 1.53 & $\mathrm{~N}(14)-\mathrm{C}(12)-\mathrm{S}(16)$ & 106 & $\mathrm{H}(25)$ & +0.16 \\
\hline $\mathrm{C}(24)-\mathrm{C}(22)$ & 1.53 & $\mathrm{C}(17)-\mathrm{C}(22)-\mathrm{S}(16)$ & 106 & $\mathrm{H}(26)$ & +0.09 \\
\hline $\mathrm{H}(25)-\mathrm{C}(23)$ & 1.08 & $\mathrm{C}(13)-\mathrm{N}(14)-\mathrm{C}(17)$ & 128 & $\mathrm{H}(27)$ & +0.10 \\
\hline $\mathrm{H}(26)-\mathrm{C}(23)$ & 1.09 & $\mathrm{~N}(14)-\mathrm{C}(17)-\mathrm{C}(18)$ & 111 & $\mathrm{H}(28)$ & +0.12 \\
\hline $\mathrm{H}(27)-\mathrm{C}(23)$ & 1.08 & $\mathrm{C}(22)-\mathrm{C}(17)-\mathrm{C}(18)$ & 114 & $\mathrm{H}(29)$ & +0.11 \\
\hline $\mathrm{H}(28)-\mathrm{C}(24)$ & 1.09 & $\mathrm{C}(17)-\mathrm{C}(18)-\mathrm{O}(19)$ & 114 & $\mathrm{H}(30)$ & +0.11 \\
\hline $\mathrm{H}(29)-\mathrm{C}(24)$ & 1.08 & $\mathrm{O}(20)-\mathrm{C}(18)-\mathrm{O}(19)$ & 123 & $\mathrm{H}(31)$ & +0.10 \\
\hline $\mathrm{H}(30)-\mathrm{C}(24)$ & 1.08 & $\mathrm{C}(17)-\mathrm{C}(18)-\mathrm{O}(20)$ & 124 & $\mathrm{H}(32)$ & +0.10 \\
\hline $\mathrm{H}(31)-\mathrm{C}(5)$ & 1.08 & $\mathrm{C}(18)-\mathrm{O}(19)-\mathrm{H}(21)$ & 108 & $\mathrm{H}(33)$ & +0.12 \\
\hline $\mathrm{H}(32)-\mathrm{C}(6)$ & 1.08 & $\mathrm{~N}(14)-\mathrm{C}(17)-\mathrm{C}(22)$ & 108 & $\mathrm{H}(34)$ & +0.10 \\
\hline $\mathrm{H}(33)-\mathrm{C}(4)$ & 1.07 & $\mathrm{C}(12)-\mathrm{S}(16)-\mathrm{C}(22)$ & 95 & $\mathrm{H}(35)$ & +0.09 \\
\hline $\mathrm{H}(34)-\mathrm{C}(1)$ & 1.08 & $\mathrm{C}(17)-\mathrm{C}(22)-\mathrm{C}(23)$ & 109 & $\mathrm{H}(36)$ & +0.16 \\
\hline $\mathrm{H}(35)-\mathrm{C}(2)$ & 1.08 & $\mathrm{~S}(16)-\mathrm{C}(22)-\mathrm{C}(23)$ & 110 & $\mathrm{H}(37)$ & +0.18 \\
\hline $\mathrm{H}(36)-\mathrm{C}(11)$ & 1.08 & $\mathrm{C}(24)-\mathrm{C}(22)-\mathrm{C}(23)$ & 110 & $\mathrm{H}(38)$ & +0.15 \\
\hline $\mathrm{H}(37)-\mathrm{C}(17)$ & 1.08 & $\mathrm{C}(17)-\mathrm{C}(22)-\mathrm{C}(24)$ & 114 & $\mathrm{~N}(39)$ & -0.43 \\
\hline $\mathrm{H}(38)-\mathrm{C}(12)$ & 1.08 & $\mathrm{~S}(16)-\mathrm{C}(22)-\mathrm{C}(24)$ & 109 & $\mathrm{H}(40)$ & +0.14 \\
\hline $\mathrm{N}(39)-\mathrm{C}(7)$ & 1.46 & $\mathrm{C}(22)-\mathrm{C}(23)-\mathrm{H}(25)$ & 112 & $\mathrm{H}(41)$ & +0.20 \\
\hline $\mathrm{H}(40)-\mathrm{C}(7)$ & 1.09 & $\mathrm{C}(22)-\mathrm{C}(23)-\mathrm{H}(26)$ & 109 & $\mathrm{C}(42)$ & +0.08 \\
\hline $\mathrm{H}(41)-\mathrm{N}(39)$ & 1.00 & $\mathrm{C}(22)-\mathrm{C}(23)-\mathrm{H}(27)$ & 110 & $\mathrm{C}(43)$ & -0.21 \\
\hline $\mathrm{C}(42)-\mathrm{N}(39)$ & 1.46 & $\mathrm{C}(22)-\mathrm{C}(24)-\mathrm{H}(28)$ & 110 & $\mathrm{C}(44)$ & -0.11 \\
\hline $\mathrm{C}(42)-\mathrm{N}(10)$ & 1.48 & $\mathrm{C}(22)-\mathrm{C}(24)-\mathrm{H}(29)$ & 110 & $\mathrm{H}(45)$ & +0.11 \\
\hline $\mathrm{C}(43)-\mathrm{C}(42)$ & 1.53 & $\mathrm{C}(22)-\mathrm{C}(24)-\mathrm{H}(30)$ & 112 & $\mathrm{H}(46)$ & +0.12 \\
\hline $\mathrm{C}(44)-\mathrm{C}(42)$ & 1.53 & $\mathrm{C}(1)-\mathrm{C}(5)-\mathrm{H}(31)$ & 120 & $\mathrm{H}(47)$ & +0.09 \\
\hline
\end{tabular}




\begin{tabular}{|l|l|l|l|l|l|}
\hline $\mathrm{H}(45)-\mathrm{C}(43)$ & 1.09 & $\mathrm{C}(5)-\mathrm{C}(6)-\mathrm{H}(32)$ & 120 & $\mathrm{H}(48)$ & +0.10 \\
\hline $\mathrm{H}(46)-\mathrm{C}(43)$ & 1.08 & $\mathrm{C}(6)-\mathrm{C}(4)-\mathrm{H}(33)$ & 119 & $\mathrm{H}(49)$ & +0.10 \\
\hline $\mathrm{H}(47)-\mathrm{C}(43)$ & 1.08 & $\mathrm{C}(2)-\mathrm{C}(1)-\mathrm{H}(34)$ & 120 & $\mathrm{H}(50)$ & +0.08 \\
\hline $\mathrm{H}(48)-\mathrm{C}(44)$ & 1.08 & $\mathrm{C}(1)-\mathrm{C}(2)-\mathrm{H}(35)$ & 120 & & \\
\hline $\mathrm{H}(49)-\mathrm{C}(44)$ & 1.08 & $\mathrm{~N}(10)-\mathrm{C}(11)-\mathrm{H}(36)$ & 109 & & \\
\hline $\mathrm{H}(50)-\mathrm{C}(44)$ & 1.09 & $\mathrm{C}(12)-\mathrm{C}(11)-\mathrm{H}(36)$ & 108 & & \\
\hline & & $\mathrm{N}(14)-\mathrm{C}(17)-\mathrm{H}(37)$ & 109 & & \\
\hline & & $\mathrm{N}(14)-\mathrm{C}(12)-\mathrm{H}(38)$ & 114 & & \\
\hline & & $\mathrm{C}(3)-\mathrm{C}(7)-\mathrm{N}(39)$ & 112 & & \\
\hline & & $\mathrm{C}(3)-\mathrm{C}(7)-\mathrm{H}(40)$ & 108 & & \\
\hline & & $\mathrm{C}(7)-\mathrm{N}(39)-\mathrm{H}(41)$ & 109 & & \\
\hline & & $\mathrm{C}(7)-\mathrm{N}(39)-\mathrm{C}(42)$ & 108 & & \\
\hline & & $\mathrm{C}(8)-\mathrm{N}(10)-\mathrm{C}(42)$ & 112 & & \\
\hline & & $\mathrm{N}(39)-\mathrm{C}(42)-\mathrm{C}(43)$ & & \\
\hline & & $\mathrm{C}(44)-\mathrm{C}(42)-\mathrm{C}(43)$ & 112 & & \\
\hline & & $\mathrm{N}(39)-\mathrm{C}(42)-\mathrm{C}(44)$ & 110 & & \\
\hline & & $\mathrm{N}(10)-\mathrm{C}(42)-\mathrm{C}(44)$ & 111 & & \\
\hline & & $\mathrm{C}(42)-\mathrm{C}(43)-\mathrm{H}(45)$ & 111 & & \\
\hline
\end{tabular}

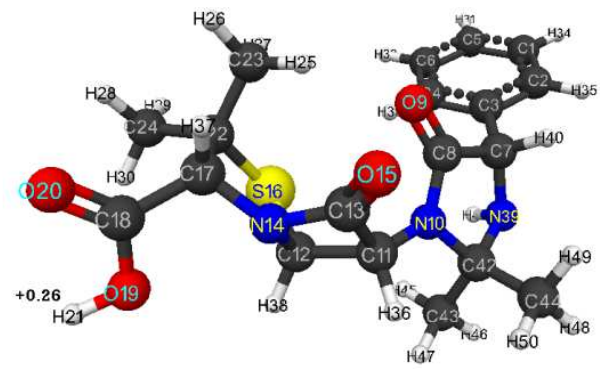

Figure 2 Geometric and electronic structure of the hetacillin molecule via DFT method $\left(\mathrm{E}_{0}=-4200142 \mathrm{~kJ} / \mathrm{mol}\right)$

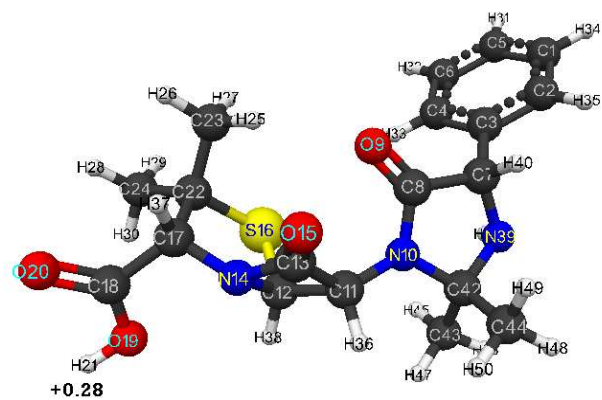

Figure 3 Geometric and electronic structure of the hetacillin molecule via AB INITIO $\left(\mathrm{E}_{0}=-4183008 \mathrm{~kJ} / \mathrm{mol}\right)$

\section{CONCLUSION}

Thus, for the first time, we performed the quantumchemical calculation of hetacillin molecule using DFTPBE0/6-311G** and AB INITIO/6-311G** methods. An optimized geometric and electronic structure of this compound was obtained. The results obtained via two methods have good convergence. Its acid strength $\mathrm{pKa}=12$ and 11 is theoretically estimated. It was found that hetacillin belongs to the class of weak $\mathrm{OH}$ acids $(9<\mathrm{pKa}<14)$.

\section{REFERENCES}

[1] V.A. Babkin, R.G. Fedunov, K.S. Minsker et al., Connection of the universal acidity index of $\mathrm{H}$-acids with the charge on hydrogen atom (AB INITIO method), Oxidat. Communicat. 25 (January 2002) 21-47.

[2] V.A. Babkin, D.S. Andreev, A.A. Pristanskov et al., The Relationship between the Antimicrobial Properties of Benzylpenicillin and the Quantum Chemical Parameters of Its Structure (The DFT Method), Amer. Chem. Sci. J. 11 (January 2016) 1-7.

[3] B.M. Bode, M.S. Gordon, MacMolPlt: A Graphical User Interface for GAMESS, J. Molec. Graphics 16 (1998) 133-138.

[4] A.A. Granovsky, Firefly version 8, 2013. Retrieved from: http://classic.chem.msu.su/gran/firefly/index.html

[5] M.W. Schmidt, K.K. Baldridge, J.A. Elbert et al., General Atomic and Molecular Electronic Structure Systems, J. Comput. Chem. 14 (1993) 1347-1363.

[6] Wikipedia contributors (31 August 2017). Hetacillin. In Wikipedia, The Free Encyclopedia. Retrieved from: https://en.wikipedia.org/ w/index.php?title=Hetacillin\&oldid=798266579 